Hybrid Monte-Carlo Method for Simulating Neutron and Photon Radiography

H. Wang, V. Tang

October 12, 2012

Nuclear Instruments and Methods in Physics Research Section A: Accelerators, Spectrometers, Detectors and Associated Equipment 
This document was prepared as an account of work sponsored by an agency of the United States government. Neither the United States government nor Lawrence Livermore National Security, LLC, nor any of their employees makes any warranty, expressed or implied, or assumes any legal liability or responsibility for the accuracy, completeness, or usefulness of any information, apparatus, product, or process disclosed, or represents that its use would not infringe privately owned rights. Reference herein to any specific commercial product, process, or service by trade name, trademark, manufacturer, or otherwise does not necessarily constitute or imply its endorsement, recommendation, or favoring by the United States government or Lawrence Livermore National Security, LLC. The views and opinions of authors expressed herein do not necessarily state or reflect those of the United States government or Lawrence Livermore National Security, LLC, and shall not be used for advertising or product endorsement purposes. 


\section{Hybrid Monte-Carlo Method for Simulating Neutron and Photon Radiography}

Han Wang ${ }^{1, \text { a) }}$ and Vincent Tang ${ }^{1, b)}$

Lawrence Livermore National Laboratory, 7000 East Ave, Livermore, $C A 94550$

(Dated: 19 October 2012)

We present a Hybrid Monte-Carlo method (HMCM) for simulating neutron and photon radiographs. HMCM uses the combination of a Monte-Carlo particle simulation for calculating incident film radiation and a statistical post processing routine to simulate film noise. Since the method relies on Monte Carlo N-Particle ${ }^{1}$ for transport calculations, it is easily generalized to most non-destructive evaluation (NDE) simulations. We verified the method's accuracy through comparison of predicted and measured equivalent penetrameter sensitivity values for x-ray radiography tests outlined in ASTM International's E592-99 Standard Guide ${ }^{2}$. Potential uses for the method include benchmarking the NDE capability of different radiography systems for applications such as radiological source replacement.

Keywords: X-ray, radiography, MCNP, neutron imaging, gamma imaging, ASTM, simulation, equivalent penetrameter sensitivity

\footnotetext{
a) Electronic mail: wang61@llnl.gov, Corresponding Author

b) Electronic mail: tang23@llnl.gov
} 


\section{INTRODUCTION}

In this paper, we describe a new capability for simulating radiographic images. The simulation consists of a Hybrid Monte-Carlo method (HMCM) that allows the entire process, from source output to film response, to be modeled at moderate computational cost. Every step after particle production is modeled by HMCM, including but not limited to linear attenuation, scatter in the test object, scatter within the film, and secondary Compton electrons. Existing techniques treat linear attenuation well but deal with scattering in three broad categories ${ }^{3}$, computationally intensive brute force Monte Carlo transport ${ }^{4,5}$, approximations based on partial calculations ${ }^{6}$ or neglection ${ }^{7,8}$. Of the three categories, the first is slow, the second has limited regimes of convergence and the third is insufficient for high fidelity applications ${ }^{3}$. In contrast with existing techniques, HMCM blends characteristics of the first and second category through a multi-fidelity model where scattering is simulated coarsely outside the film packet but minutely within. Additionally, HMCM supports both neutron and photon sources, and evaluates the spatial and energy film response for the fully coupled photon-electron problem. The latter allows for realistic film responses since in a typical film package, the film is sandwiched between lead intensifier sheets that produce significant number of Compton electrons per incident photon. Finally, HMCM relies on the proven framework of Monte Carlo N-Particle ${ }^{1}$ (MCNP) transport code for particle simulations to provide adaptability to a wide range of NDE simulations ${ }^{9-11}$.

An important application of simulated radiography is its use in comparing and evaluating different radiography systems. ASTM International (ASTM) has standardized guidelines for evaluating the effectiveness of a radiography system through the use of the equivalent penetrameter sensitivity (EPS) metric ${ }^{2,12}$. The EPS metric is defined as Eqn. 1 and quantifies the smallest feature size that can be seen by a radiography system. ASTM provides a set of tests for determining the system's EPS which consists of imaging parallel penetrameters on top of a steel plate, Figure 1. The penetrameters have arrays of holes indicating different EPS values and the EPS value of the system is determined by the last visible line. We have simulated this process using HMCM for a standard x-ray setup and compared it with the EPS data provided by ASTM. Figure 2 shows the EPS comparison between our model and ASTM experimental results for different steel plate thicknesses and film types. The match is excellent for D2 and D4 film. For D8 film, we were unable to obtain the required film data 
so we simulated D7 film instead. As expected, D7 demonstrated slightly lower EPS values while following the shape of the D8 curve.

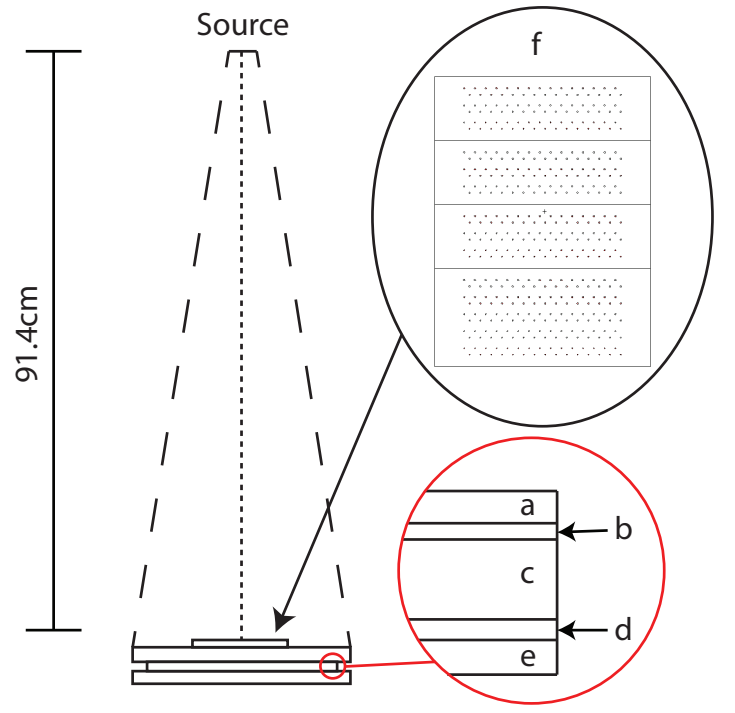

FIG. 1. ASTM testing setup. The various film components are a) front lead intensifier, b) front grain emulsion, c) adhesive substrate, d) back grain emulsion, e) back lead intensifier. f) is a top down view of the penetrameter plates.

$$
\mathrm{EPS}=\frac{100}{x} \sqrt{\frac{t \times h}{2}}
$$

s.t. $x$ is the thickness of the plate,

$t$ is the thickness of the penetrameter,

and $h$ is diameter of the hole

We now describe the model and the EPS testing procedure in full detail. Section II presents an overview of the entire algorithm. Section II A through IID shows the various sub-models of the algorithm in order of their usage. Section III A reviews ASTM testing procedures and Section III B presents numerical results. Finally, Section IV wraps up with conclusions.

\section{ALGORITHM OVERVIEW}

Our hybrid Monte-Carlo algorithm proceeds in four parts, each simulating a different portion of the the imaging setup at a different level of detail, Figure 3. Each submodel is 


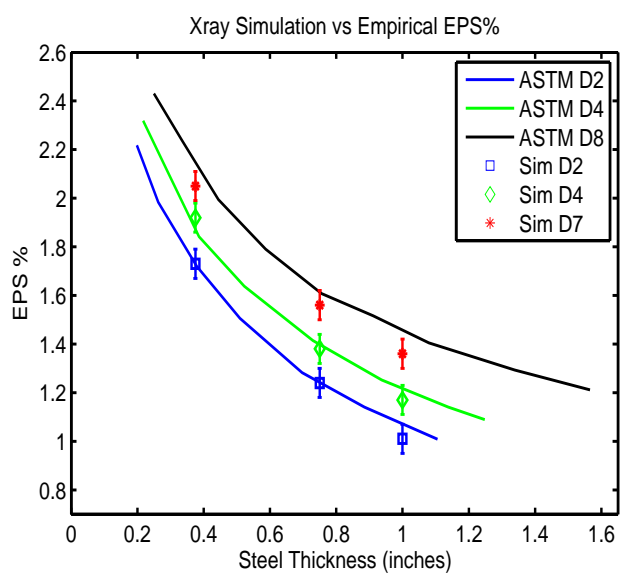

FIG. 2. Equivalent Penetrameter Sensitivity measurements from ASTM vs measurements from HMCM. D8 film data was unavailable so D7, a film with nearly the same speed, was used as a surrogate.

gridded into pixels of the same size with the exception of the scattering model.

First, we simulate the radiation incident on the film after accounting for attenuation. This portion of the algorithm only uses ray tracing and we call it the direct contribution model (DCM).



FIG. 3. Flowchart of HMCM, note that the direct contribution model and the scattering model can be executed in parallel.

Next, we simulate the radiation deposited onto the film with attenuation and scatter. 
This second step is a low fidelity analogue simulation using Monte-Carlo particles. We denote this step the scattering model (SM).

Then we analyze the data from the DCM model and average the energy bins over all pixels to form an energy spectrum. We take this hardened spectrum and simulate an intrafilm response model (FRM). The goal of this model is to find the energy deposited in pixel $j$ given a particle incident in pixel $i$.

Afterwards, we combine the FRM and DCM together to estimate the energy deposited in each pixel due to direct contribution and realistic intra-film scattering, we term this result the intermediate image. Next the difference between the mean dose per $\mathrm{cm}^{2}$ of the intermediate image and the mean dose per $\mathrm{cm}^{2}$ of the $\mathrm{SM}$ is found and added to each pixel in the intermediate image. This step serves to lower contrast, and we call the sum the net dose image.

As shown by prior work ${ }^{13-15}$, optical density can be modeled as a function of dose. Thus, the net dose image is converted to optical density and noise is added to the the optical density by taking into account the number of film grains per pixel. This is the film noise model (FNM).

Finally, this optical density image is processed through Eqn. 2 to retrieve the final simulated image.

$$
W_{i, j}=\frac{1}{10^{D_{i, j}}}
$$

Here, $W_{i, j}$ denotes the fraction of light which will pass through pixel $i, j$ and $D_{i, j}$ denotes the optical density with noise in pixel $i, j$.

\section{A. Direct Contribution Model}

The direct contribution model factors in the linear attenuation of source flux through the test object before reaching the film packet. The main purpose of this model is to resolve the

probability that a particle emitted by the source will become incident on a specific pixel in the film packet and to determine the spectrum of incident radiation on the film packet after hardening by the test object.

We implemented this submodel in MCNP because of MCNP's widespread availability and its proven accuracy in calculating particle transport and flux attenuation ${ }^{9-11}$. MCNP 
is run in mode " $\mathrm{n}$ " for neutron sources and mode "p" for photon sources. If both particle types are present, two runs, one of each particle type, are executed. In MCNP, we used f5 FIR (array of point detectors for planar radiograph flux image) tallies with discrete energy bins which bracket the source's emission spectrum. The FIR tallies are constructed in a rectangular array with each pixel being $150 \times 150 \mu \mathrm{m}$. We iterated through various pixel sizes but decided on square $150 \mu \mathrm{m}$ pixels because it was a good compromise between resolution and computation complexity. We refer to $N_{i, j, k}$ as the count of particles in energy bin $k$ in pixel $i, j$.

In addition to the hardened spectrum, we can also infer the probability that a particle of any energy will cross pixel $i, j$. We call this value $U_{i, j}$ and define it as the probability incident matrix, Eqn. 3, Figure 4:

$$
U_{i, j}=\frac{\sum_{k} N_{i, j, k}}{\sum_{i} \sum_{j} \sum_{k} N_{i, j, k}}
$$

\section{B. Scattering Model}

The scattering model is an analogue model of the entire simulation domain, which itself is divided into a high fidelity portion, the film packet, and a low fidelity portion, everything else.

In the high detail portion, the film packet is further subdivided into its components of front lead intensifier, front film grain emulsion layer, adhesive substrate, back film grain emulsion layer, and back lead intensifier, Figure 5.

While we opt for a detail model of the film packet to capture the exact energy deposited on the film grain layers, the geometry of the test object is simplified. This is justified because this step is only used to find the mean energy deposited into the grain layers and each grain layer is modeled as a single energy deposition tally. Thus, the loss of fidelity in local variations in the SC model justifies the simplification of test object geometry.

To aide in the intrinsically subjective step of test object geometry simplification, we offer the following guidelines:

1. Merge features which have similar materials and densities. Create one feature with blended material composition and the mean density. 


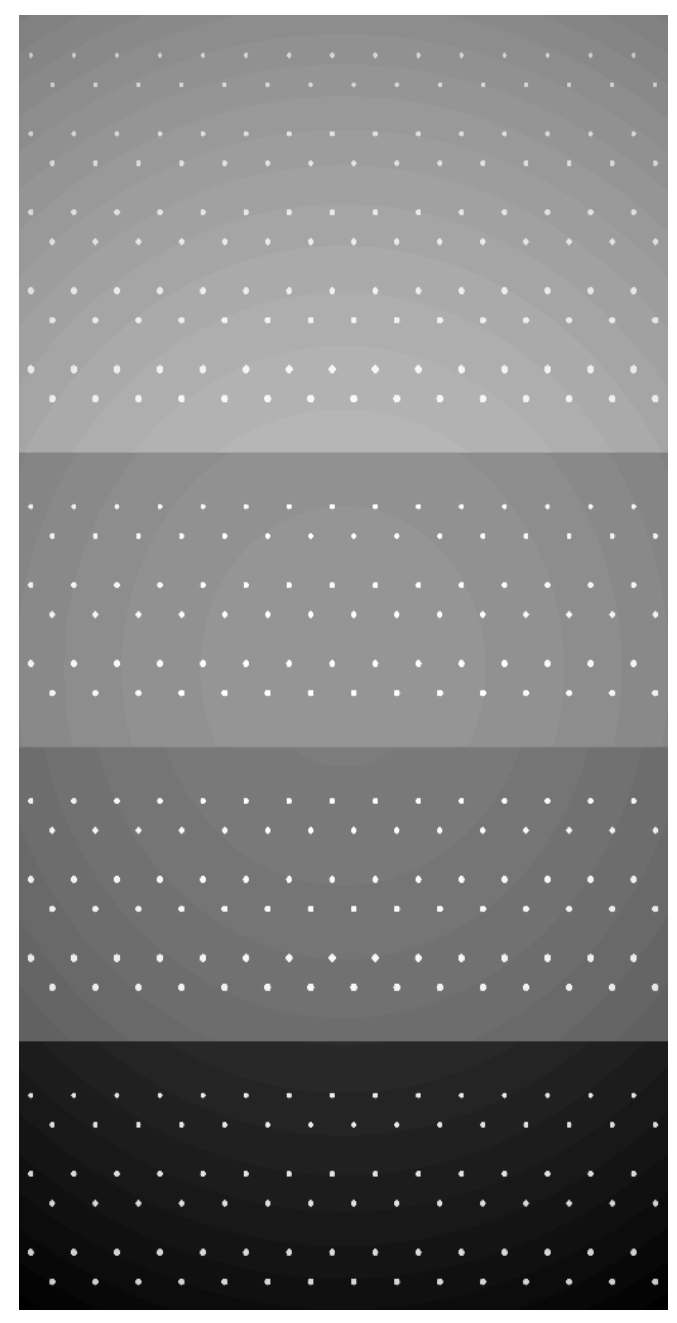

FIG. 4. A sample U matrix of an ASTM penetrameter on top of a $3 / 4$ " steel plate for a $264 \mathrm{kV}$ x-ray source. Note the sharp edges of the features.

2. Remove small interior features of low volume relative to the test object.

3. Smooth exterior faces and remove small features (i.e. dimples on a golf ball)

In the end, preserve as much detail as computational resources allow, iterating through the guideline as necessary.

In practice, we once again use $\mathrm{MCNP}^{1}$, for the $\mathrm{SM}$ sub-model. For neutron sources, MCNP is run in "n,p,e" mode and for photon sources, MCNP is run in "p,e" mode. For joint sources, two runs are executed like in the DCM.

In addition to creating the necessary cells for the simplified test object, we modeled each component of the film packet as its own cell. Finally, we attached a "*f8:p,e" (photon) or 


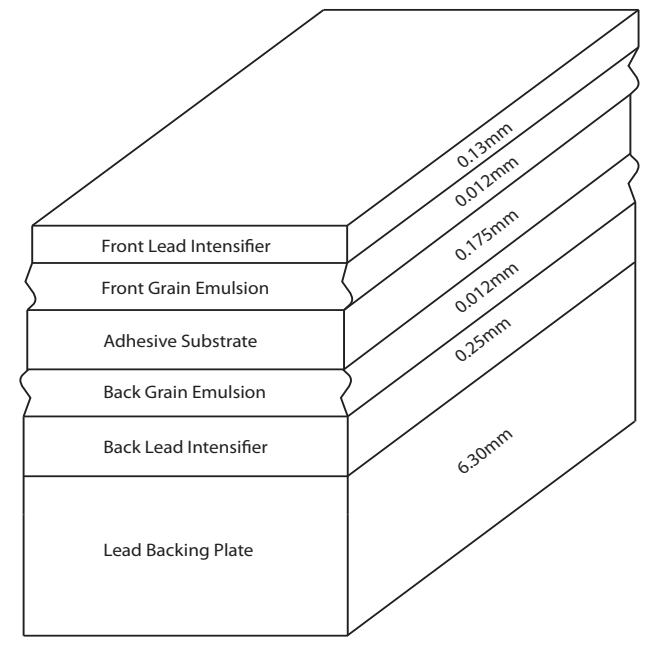

FIG. 5. Cross-sectional slice of a standard film packet, notice how thin the actual grain layers are in relation to the entire film. Figure is not to scale.

both a "f6:n" and "*f8:p,e" (neutron) energy deposition tally to the front and back film grain layer to capture the expected amount of total energy deposited on each grain layer per source particle. For neutron sources, "f6:n" tally values are multiplied by the weight of the grain layer and then added to their "*f8:p,e" counterpart for the total deposited energy. For photon sources, the grain layer energy is just the "*f8:p,e" value. The net energy deposited to the grain layers, $\gamma$, is the sum of the values of the two grain layer energies. This amount contains the total energy deposited, including scattering effects.

\section{Film Response Model}

The film response model is innately coupled with the DCM model because it requires the shifted emission spectrum that is incident to the film packet. This spectrum is calculated in the following manner. Let $N_{i, j, k}$ denote the $\mathrm{k}^{\text {th }}$ energy bin of pixel $i, j$. Now, we can calculate $P_{k}$, the probability of a particle in the $\mathrm{k}^{\text {th }}$ energy bin making it to the film packet, via Eqn. 4.

$$
P_{k}=\frac{\sum_{j} \sum_{i} N_{i, j, k}}{\sum_{j} \sum_{i} \sum_{k} N_{i, j, k}}
$$

While Eqn. 4 only holds for a homogeneous test object spanning a small solid angle of the source, this approximation is still closer, on average, to the film's incident radiation 
spectrum than the source's emission spectrum. Possible ways to increase the fidelity and localize $P_{k}$ to each pixel are proposed in the future work section.

After finding the $P_{k}$ 's, we interpolate the bins to create the continuous incident particle energy spectrum, $I(e)$, Eqn. 5.

$$
\begin{gathered}
I(e)=\frac{P_{k}^{+}+\left(e-P_{k}^{+}\right) \Delta P_{k+1}}{\int_{e} I(e)} \\
\text { s.t. } \Delta P_{k+1}=P_{k+1}^{-}-P_{k}^{+}
\end{gathered}
$$

and $\Delta P_{k}^{-}, \Delta P_{k}^{+}$are respectively the left

and right edges of bin $P_{k}$

Using the spectrum defined by Eqn. 5, we create a detailed film packet model outlined in Figure 5 spanning $15 \times 15$ pixels. Instead of creating one energy tally for each film grain layer, we further discretize each layer with a $15 \times 15$ grid. This creates film grain voxels, volumetric pixels, in each grain layer which are bijectively mapped to each DCM pixel, Figure 6 . The grid size of $15 \times 15$ was chosen because we found that past 6 pixels in each direction, the intra-film scattering becomes negligible. Since this finding is specific to our defined pixel size of $150 \times 150 \mu m$, the optimal grid size will change with pixel size.

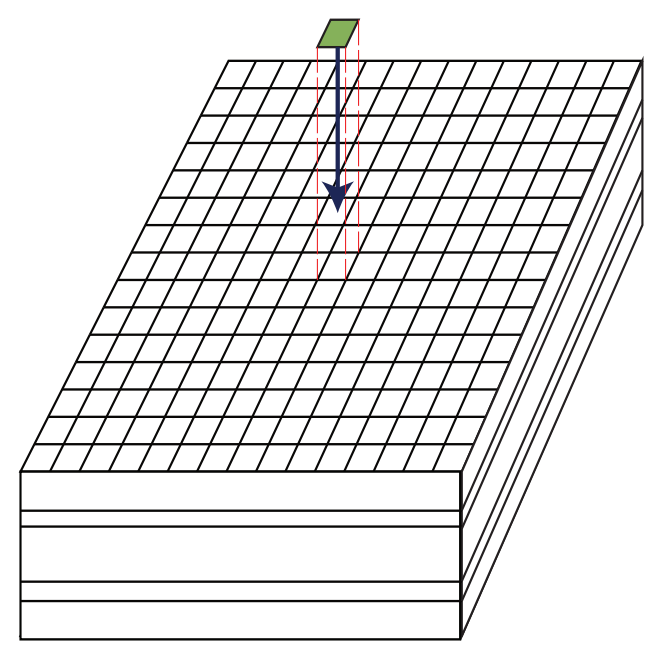

FIG. 6. Film response model simulation domain

Over the center pixel, we define an uniform source in MCNP which is equivalent in size to one pixel and only emits particles perpendicular to the film packet's plane, Figure 6 . The main reasoning behind using a square pixel source is the output of the energy pulse tallies is exactly $e_{i, j}$, the energy deposited on pixel $j$ given a particle incident on pixel $i$. Thus, 
we can concatenate the results of the front and back energy pulse tallies spacially into two matrices, $F$, and $B$, where $F_{i, j}$ denotes the front energy tally from row $i$ and column $j$, Figure 7. Then, $R$, the direct contribution energy to each pixel with intra-film scattering, is the convolution of the probability incident matrix, $U$, with $(F+B)$.

Similar to SM, neutron sources use both "f6:n" and "*f8:p,e" tallies while photon sources use only "*f8:p,e" tallies. Joint sources are run separately and all "f6:n" tallies are multiplied by the weight of the grain layer in a pixel before addition to their "*f8:p,e" counterparts.

$$
\begin{array}{r}
R_{n}=U_{n} \star\left(F_{n}+B_{n}\right) \\
R_{p}=U_{p} \star\left(F_{p}+B_{p}\right)
\end{array}
$$

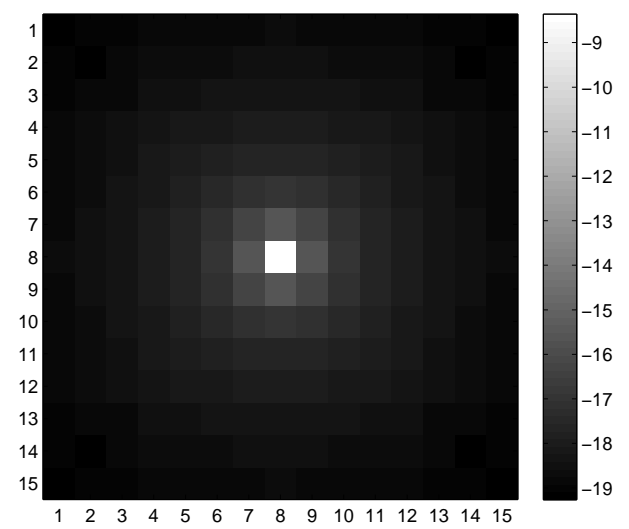

FIG. 7. $\log (F+B)$ matrix from a $3.5 \mathrm{MeV}$ neutron source

\section{Film Noise Model}

The film noise model accomplishes three things. First, it merges the particle specific $R_{n / p}$ and $\gamma_{n / p}$ together into a joint $R$ and $\gamma$. Second, it combines $R$ and $\gamma$ together to create the radiation dose per pixel, $T$. Finally, it seeks to post process $T$ into an image seen on a view box based on properties of the film used.

All MCNP tally outputs are normalized to per source particle. Thus for a joint source which emits $t$ neutrons per photon, $R_{n / p}$ and $\gamma_{n / p}$ can be merged through Eqn. 7 .

$$
R=\frac{1}{t+1}\left[t \times R_{n}+R_{p}\right], \gamma=\frac{t \times \gamma_{n}+\gamma_{p}}{t+1}
$$


Next to find the dose per pixel, we first convert $R$ and $\gamma$ to rads.

$$
\begin{aligned}
R_{\text {rad }} & =\frac{1.602 \times 10^{-} 8 \times R}{\text { Weight of grains per pixel (grams) }} \\
\gamma_{\text {rad }} & =\frac{1.602 \times 10^{-} 8 \times \gamma}{\text { Weight of grains in film (grams) }}
\end{aligned}
$$

Next, we find $r_{r a d}=\operatorname{mean}\left(R_{\text {rad }}\right)$ which is the mean dose per pixel. Then, we normalize both $r$ and $\gamma$ to $\frac{\mathrm{rad}}{\mathrm{cm}^{2}}$ and find the difference between them, $\beta=\gamma-r$. $\beta$ is, on average, the increased dose per $\mathrm{cm}^{2}$ when accounting for scattering in the simulation domain. Now, we can solve for the radiation dose per pixel with scattering though Eqn. 8:

$$
T=R_{\text {rad }}+\beta \times(\text { Pixel Size })
$$

Given, $T$, we now wish to generate an image statistically equivalent to a physical film exposed to our simulation conditions, developed and placed on a view box. The first step in linking $T$ to the view box image is the conversion of rad to optical density.

While the dose to optical density relationship is bijective, the function is nonlinear and we use the data provided by Gray ${ }^{13}$ and create a linear interpolation function $f\left(T_{i, j}\right): \rightarrow O_{i, j}$ as our approximate mapping from rad to optical density, $O$.

ASTM guidelines call for a film to be exposed to until the optical density averaged over the entire film is equal to $2^{2}$. This means $T$ would need to be scaled to match the expected mean optical density. Since MCNP normalizes all outputs to per source particle, $T$ is actually in units of $\frac{\mathrm{rad}}{\text { particle }}$. Thus, $T$ needs to be multiplied by $\alpha$, the number of particles required to reach a mean optical density of 2 . Given $f, \alpha$ can be found by solving Eqn. 9 .

$$
\alpha=\frac{f^{-1}(2)}{\operatorname{mean}(T)}
$$

Then, optical density for pixel $i, j$ can be found via Eqn. 10 .

$$
O_{i, j}=f\left(\alpha T_{i, j}\right)
$$

The next step in this process is to fold in the noise which result from the random activation of film grains within the grain layer. A statistical model which does account for grain activation is the binomial model, ${ }^{16}$. This model assumes that in each pixel, there is a finite number of grains, $N_{0}$ and for a given dose uniform over the pixel, each grain has a probability 
$\eta$ of activating. This implies the actual number of activated grains should follow a binomial distribution, Eqn 11, and there is a limit to optical density. This limit, $O_{\max }$, is achieved when all grains are activated, Eqn 12.

$$
\begin{gathered}
P_{n}=\frac{N_{0} !}{n !\left(N_{0}-n\right) !}(1-\eta)^{\left(N_{0}-n\right)} \eta^{n} \\
O_{\max }=a m \eta \log _{10}(e)
\end{gathered}
$$

s.t. $a$ is the area of each grain,

$m$ is the total number of grains per unit area

and $e$ is Euler's number

Assuming uniform grain size, a fixed $O_{\max }$ and a grain overlap factor of $\nu$, where $\nu=0$ means complete overlap and $\nu=1$ means zero overlap (Figure 8), the optical density can be related to the activation probability through Eqn. 13.

$$
O=\eta^{\nu} O_{\max }
$$

Futhermore, $O$ itself can be modeled as a normal random variable with sigma as defined in Eqn. 14.

$$
\begin{gathered}
\sigma_{O}=\frac{1}{\beta \sqrt{N_{0}}} \sqrt{O^{2-\beta}\left(O_{\max }^{\beta}-O^{\beta}\right)} \\
\text { where } \beta=\frac{1}{\nu}
\end{gathered}
$$

In order to incorporate grain statistical noise, we define $D$, optical density with grain noise, by sampling with respect to Eqn. 15.

$$
D_{i, j} \sim N\left(O_{i, j}, \sigma_{O_{i, j}}\right)
$$

Given $D$, we can compile $W$, the final image and the simulated equivalent of a film over a view box (Figure 9), according to Eqn. 16.

$$
W_{i, j}=\frac{1}{10^{D_{i, j}}}
$$




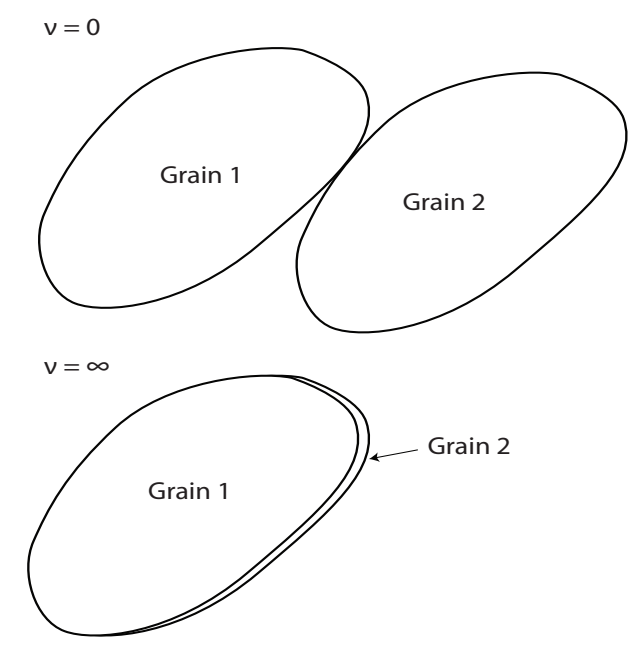

FIG. 8. Grain overlap characterized by $\nu$

\section{NUMERICAL RESULTS}

In this section, we present ASTM relative image quality response (RIQR) testing procedures and how we recreated the procedures in HMCM.

\section{A. Relative Image Quality Response Testing Guidelines}

ASTM International presents two standards which use EPS values, E746 ${ }^{12}$ and E592 . While both standards are similar in methodology, E746 stipulates that EPS values be calculated at $50 \%$ hole visibility while E592 requires $100 \%$ hole visibility. The former is a set of general guidelines for using EPS values and penetrameters for comparing different radiography systems while the latter provides a set of EPS values for x-ray tube based radiography of steel plates with various thicknesses using different speed films. Since we used E592 as our verification dataset, we adhered to the methodology outlined in E592 and first describe the experimental setup below.

The first step in the x-ray EPS tests outlined in E592 was to fix the x-ray tube source $914 \mathrm{~mm}$ away and perpendicular to a film packet. Next, a plate of homogeneous rolled steel was placed in front of the film packet. Then on top of the steel plate, penetrameters of varying thickness and holes sizes were laid. Assuming the same penetrameter as in E746, the penetrameters were arranged such that hole EPS values increased from top to bottom. 


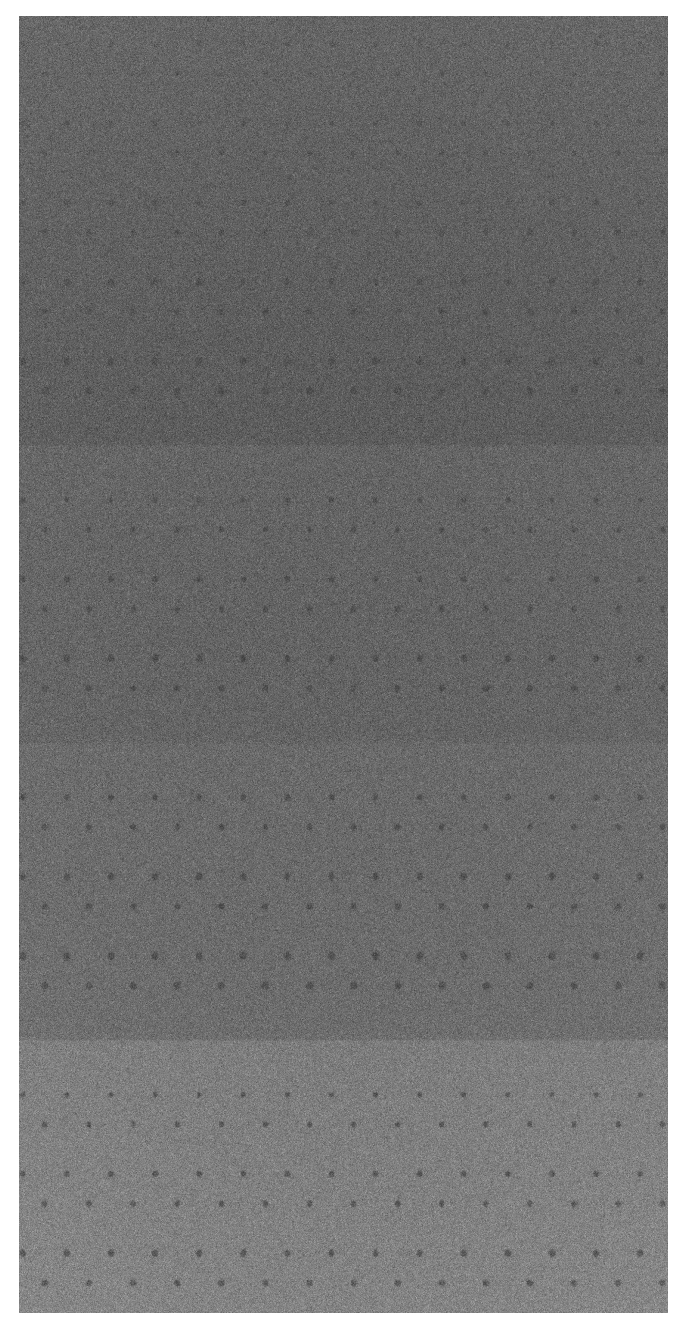

FIG. 9. Final Image of ASTM benchmark penetrameter for 3/4" plate and D4 film. The x-ray tube voltage was $264 \mathrm{kV}$ for a $1200 \mathrm{~mA}$-sec exposure to reach an optical density of 2 . EPS values range from $0.95 \%$ (top) to $1.93 \%$ (bottom).

The full setup is shown in Figure 1.The EPS value was calculated for each row of holes on the penetrameter according to Eqn. 1.

The x-ray tube was turned on and the film was exposed until a mean optical density of 2. The film was then placed on a view box for evaluation. For the E592 dataset, the x-ray tube voltage was varied for the setups with different plate thicknesses and films to achieve a constant exposure time of $1200 \mathrm{~mA}-\mathrm{sec}$.

During the evaluation process, the relative image quality response of the system was defined as the lowest EPS value where the holes were visible with $100 \%$ certainty. This number quantified the performance of the tested x-ray radiography system for a particular 
set of plate thickness, film package, and x-ray tube voltage.

Since slower films have less grain noise, they produce lower EPS values than their faster counterparts. Figure 2 shows our simulated and the measured EPS values for the x-ray radiography tests laid out in E592. The match is excellent. We now review the details of these simulations.

\section{B. Simulation Details and Results}

In our simulation, we generated images for D2, D4, and D7 film at plate thicknesses of 3/8", 3/4" and 1". Unfortunately, we were not able to obtain dose to optical density data for D8 which prevented us from making D8 predictions for direct comparison with the ASTM E592 D8 data.

To produce these simulations, we first used two tools to find the emission spectrum of the x-ray tube. For voltages lower than $300 \mathrm{kV}$, we used Spekcalc, a program for calculating the $\mathrm{x}$-ray emission spectra from tungsten anodes ${ }^{17}$. For voltages greater than $300 \mathrm{kV}$, we used MCNP in coupled photon-electron mode.

Since E592 necessitated that imaging be done at constant time with voltage variations ${ }^{2}$, we needed to figure out the voltage at which the X-ray tubes were run at for the corresponding emission spectrum. For the tube voltage, we calculated the required voltage to expose the film to a mean optical density of 2 in 1200 seconds given $1 \mathrm{~mA}$ of current. The corresponding voltages are listed in Table I.

For the simulation, we modeled the steel plate as rectangular prism of SS304 steel with a density of $8 \frac{\mathrm{gm}}{\mathrm{cm}^{3}}$ and the penetrameters as $8 \frac{\mathrm{gm}}{\mathrm{cm}^{3}} \mathrm{SS} 304$ steel rectangular prisms with cylindrical holes. In the DCM submodel, we used twenty energy bins per pixel and in the SM submodel, we simplified the penetrameter geometries by removing the cylindrical holes. For the film noise model, we used dose to optical density from ${ }^{13}$ and film grain data given by Segal et al. ${ }^{16}$ and Lanier et al. ${ }^{18}$ which is presented in Table II.

We ran the MCNP portions of the model on a 192 core cluster and the walltime for DCM, SM, and FR were 20 minutes, 15 minutes, and 5.6 minutes respectively. The FNM model took 60 seconds on a desktop. The EPS values from the simulations are plotted against the values supplied by ASTM in Figure 2 and they agree well. 
TABLE I. Voltages used in calculating emission spectrum given plate thickness and film type

\begin{tabular}{ccc}
\hline \hline Plate Thickness & Film Type & Tube Voltage $(\mathrm{kV})$ \\
\hline $3 / 8 "$ & $\mathrm{D} 2$ & 240 \\
\hline $3 / 8 "$ & $\mathrm{D} 4$ & 185 \\
\hline $3 / 8 "$ & $\mathrm{D} 7$ & 152 \\
\hline $3 / 4 "$ & $\mathrm{D} 2$ & 336 \\
\hline $3 / 4 "$ & $\mathrm{D} 4$ & 264 \\
\hline $3 / 4 "$ & $\mathrm{D} 7$ & 212 \\
\hline $1 "$ & $\mathrm{D} 2$ & 365 \\
\hline $1 "$ & $\mathrm{D} 4$ & 312 \\
\hline $1 "$ & $\mathrm{D} 7$ & 250 \\
\hline \hline
\end{tabular}

TABLE II. Film parameters used in film noise model

\begin{tabular}{cccc}
\hline \hline Film Type & $a\left(\mu m^{2}\right)$ & $m\left(\frac{\text { grains }}{\mu m^{2}}\right)$ & $\beta$ \\
\hline D2 & 3.6 & 2 & 1.30 \\
\hline D4 & 7.10 & 0.9 & 1.33 \\
\hline D7 & 12.6 & 0.6 & 1.37 \\
\hline \hline
\end{tabular}

\section{CONCLUSION}

We presented and validated a new method for simulating radiographs. The method is general in that it can handle neutron and photon sources. In addition, the method accounts for linear attenuation, scattering, energy diffusion within the film, and grain noise effects. Overall, the method is predictive and would be useful for evaluating the effectiveness of different imaging systems for applications such as radiological source replacement.

\section{ACKNOWLEDGMENTS}

We thank J. McCarrick, J. Hall, and B. Rusnak for useful discussions. This work was supported by the US. Department of Energy Na-22 Office of Nonproliferation Research and Development under the Radiological Source Replacement program and was performed under 
the auspices of the U.S. Department of Energy by Lawrence Livermore National Laboratory under Contract DE-AC52-07NA27344.

\section{REFERENCES}

${ }^{1}$ Radiation Safety Information Computational Center, MCNP User Manual, Version 5, Los Almos National Laboratory (2003).

${ }^{2}$ ASTM Standard E592-99, “ASTM E592-99 Standard Guide to Obtainable ASTM Equivalent Penetrameter Sensitivity for Radiography of Steel Plates .25 to 2 in. (6 to $51 \mathrm{~mm}$ ) Thick with X Rays and 1 to 6 in. (25 to $152 \mathrm{~mm}$ ) Thick with Cobalt-60," (1999).

${ }^{3} \mathrm{~F}$. Inanc, "Scattering and its role in radiography simulations," NDT \& E International 35, $581-593(2002)$.

${ }^{4}$ M. Winslow, X. G. Xu, and B. Yazici, "Development of a simulator for radiographic image optimization," Computer Methods and Programs in Biomedicine 78, 179 - 190 (2005).

${ }^{5}$ A. Bonin, B. Lavayssiere, and B. Chalmond, "Moderato: A monte-carlo radiographic simulation," Tech. Rep. (Advanced Materials, Manufacturing, and Testing Information Analysis Center, 1999).

${ }^{6}$ J. Gray, J. Xu, S. Wendt, T. Jensen, and F. Inanc, XRSIM Users Manual, Center for Nondestructive Evaluation: Iowa State University (2011).

${ }^{7}$ S. M. Kang, C.-H. Shin, P. N. V. Ha, C. I. Chol, J. K. Kim, and Y. K. Kim, "Comparison to images from a computed radiography system for non-destructive testing using selenium75, iridium-192, and x-rays," Journal of Korean Physical Society 59, 717 (2011).

${ }^{8}$ J. Xu, J. Wallingford, R. M. Jensen, T. Gray, and J. N., "Recent developments in the x-ray radiography simulation code: Xrsim," Tech. Rep. (Advanced Materials, Manufacturing, and Testing Information Analysis Center, 1994).

${ }^{9}$ A. C. Miller, Jr., J. L. Cochran, and V. E. Lamberti, "Monte carlo modeling of high-energy film radiography," Tech. Rep. (Oak Ridge Y-12 Plant, 2003).

${ }^{10}$ J. M. Boone, M. H. Buonocore, and I. Virgil N. Cooper, "Monte carlo validation in diagnostic radiological imaging," Medical Physics 27, 1294-1304 (2000).

${ }^{11}$ J. V. Siebers, P. J. Keall, B. Libby, and R. Mohan, "Comparison of egs4 and mcnp4b monte carlo codes for generation of photon phase space distributions for a varian 2100c," Physics in Medicine and Biology 44, 3009 (1999). 
${ }^{12}$ ASTM Standard E746, “ASTM E746-07 Standard Practice for Determining Relative Image Quality Response of Industrial Radiographic Imaging Systems," (2007).

${ }^{13}$ J. Gray, "Final report for nasa grant/cooperative agreement nag-i-1933," Tech. Rep. (Center for NDE, Applied Sciences Complex, Iowa State University, 1998).

${ }^{14}$ A. Schumm and U. Zscherpel, "Using the en584-1 film characterization in radiographic modelling," in International Symposium on Digital industrial Radiology and Computed Tomography (2007).

${ }^{15}$ T. Jensen, T. Aljundi, J. N. Gray, and R. Wallingford, "A model of x-ray film response," Review of Progress in Quantitative Nondestructive Evaluation 15, 441-448 (1996).

${ }^{16}$ Y. Segal, D. Ingman, and Y. Bushlin, "Noise and granularity in radiographs," Materials Evaluation 46, 513-517 (1988).

${ }^{17}$ G. Poludniowski, G. Landry, F. DeBlois, P. M. Evans, and F. Verhaegen, "Spekcalc : a program to calculate photon spectra from tungsten anode x-ray tubes," Physics in Medicine and Biology 54, N433 (2009).

${ }^{18}$ N. E. Lanier, J. S. Cowan, and J. Workman, "Characterization and cross calibration of agfa d4, d7, and d8 and kodak sr45 x-ray films against direct exposure film at 4.05.5kev," Rev. Sci. Instrum. 77, 435 (2006). 